\title{
Sex differences in thigh muscles characteristics: a systematic review
}

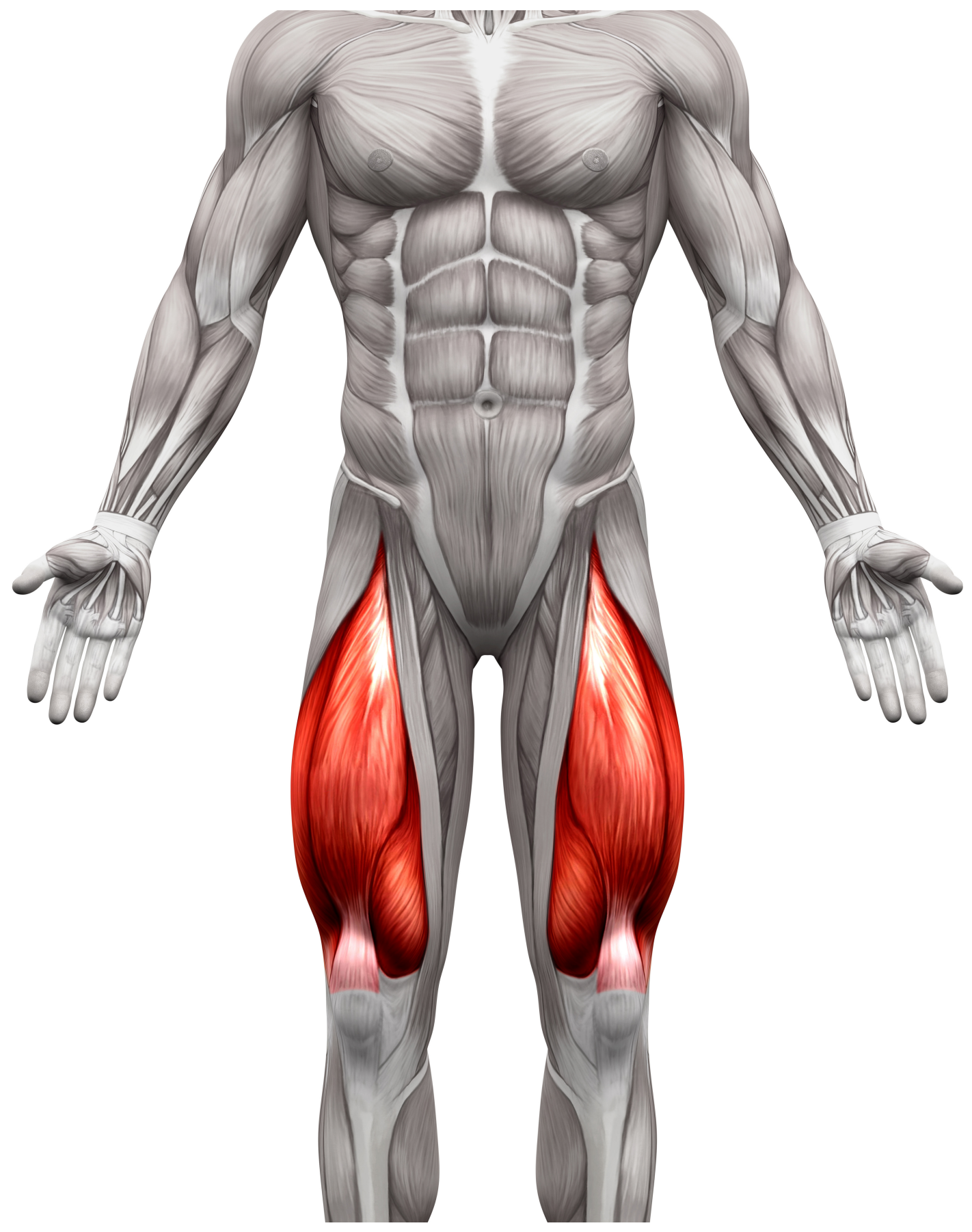




\section{Connan $\mathrm{P}^{1}$, Ravelojaona $\mathrm{M}^{1}$, Grémeaux $\mathrm{V}^{2},{ }^{3}$, Rossi $\mathrm{J}^{1}$, Edouard $\mathrm{P}^{1,2,4,5}$}

${ }^{1}$ Inter-university Laboratory of Human Movement Science (LIBM EA 7424), University of Lyon, University Jean Monnet, F-42023. Saint-Etienne, France

${ }^{2}$ Swiss Olympic Medical Center, Division de médecine physique et réadaptation, CHUV, 1011 Lausanne

${ }^{3}$ Institute of Sport Sciences, Faculty of Biology and Medicine, University of Lausanne, Lausanne, Switzerland

${ }^{4}$ Department of Clinical and Exercise Physiology, Sports Medicine Unity, University Hospital of SaintEtienne, Faculty of medicine, Saint-Etienne. France

${ }^{5}$ Medical Commission, French Athletics Federation (FFA), Paris France

\section{Abstract}

Objective: To determine differences between male and female subjects in the thigh muscles characteristics, separated into architectural (pennation, thickness, and/or fascicle length), mechanical (mass, strength, power, and/or stiffness), neuromuscular (activity) and fatigue aspects, in order to better understand the sex-related differences in the risk of muscle injuries.

Methods: A systematic literature search on Pubmed was performed with different keywords: skeletal muscle AND sex characteristics AND muscle contraction, with the following limits: humans and adults (19-44 years old). Studies dealing with hamstring and quadriceps muscles, in physiological condition, and comparison between male and female healthy adult subjects were included. Studies dealing with other skeletal muscles, injuries or physiopathology situation were excluded. Thigh muscular architectural, mechanical, neuromuscular and fatigue characteristics have been analysed to determine differences between male and female subjects.

Results: Seventeen studies were included, reporting significant sex-related differences for thigh muscles architecture and mechanical characteristics and muscle fatigue, and especially quadriceps, while for thigh muscles neuromuscular characteristics the results were not consensual, and few information was available regarding hamstring muscles.

Conclusions: Sex-related differences in thigh muscles characteristics, and especially quadriceps, have been reported for mechanical characteristics and muscle fatigue, while for neuromuscular characteristics sexrelated differences were found to be moderate. Although several macroscopic muscle characteristics have been reported to be different between male and female healthy adult subjects, it is difficult to conclude on its exact relationship with higher muscle injury rates reported in male athletes during international athletics championships.

\section{Résumé}

Objectif: Déterminer les différences au niveau des caractéristiques des muscles de la cuisse, concernant les éléments architecturaux (pennation, épaisseur et/ou longueur du fascicule), mécaniques (masse, force, puissance et/ou rigidité), neuromusculaires (activité), et les aspects liés à la fatigue, entre les sujets masculins et féminins, afin de mieux comprendre les différences de risque musculaires liées au sexe. Méthodes: Une recherche bibliographique systématique sur Pubmed a été réalisée avec différents mots- 
clés: skeletal muscle AND sex characteristics AND muscle contraction, avec les limites suivantes: humans and adults (19-44 years old). Les études ont été inclus si elles portaient sur; les muscles ischio-jambiers et quadriceps, dans un état physiologique sain, de sujets adultes en bonne santé, avec une comparaison entre hommes et femmes. Les études portant sur d'autres muscles squelettiques, analysant des situations de blessures ou autres situations physiopathologiques ont été exclues. Les caractéristiques architecturales, mécaniques, neuromusculaires et de fatigue musculaire des muscles de la cuisse ont été analysées pour déterminer les différences entre les hommes et les femmes.

Résultats: Dix-sept études ont été incluses, rapportant des différences significatives liées au sexe pour l'architecture et les caractéristiques mécaniques de la cuisse et la fatigue musculaire, en particulier le quadriceps, tandis que les résultats portant sur les caractéristiques neuromusculaires des muscles de la cuisse n'étaient pas consensuels, et peu d'informations étaient disponibles concernant les muscles ischiojambiers.

Conclusions: Des différences liées au sexe dans les caractéristiques des muscles de la cuisse, et en particulier des quadriceps, ont été rapportées pour les caractéristiques architecturales, mécaniques et la fatigue musculaire, tandis que pour les caractéristiques neuromusculaires les différences liées au sexe se sont révélées modérées. Bien que plusieurs caractéristiques musculaires macroscopiques soient différentes entre les hommes et les femmes adultes sains en bonne santé, il est difficile de tirer des conclusions exactes quant à sa relation avec les taux plus élevés de blessures musculaires signalés chez les athlètes masculins lors des championnats internationaux d'athlétisme.

\section{Conflict of interest: No potential conflict of interest declared}

Funding: No funding

Ethical statement: Since the present systematic review did not involve human subjects the Ethical Approval or subjects' informed consent was not necessary, but we used data from previous studies, which followed the Ethical standard for research on human subjects according to the Declaration of Helsinki.

\section{Introduction}

The practice of athletics (track and field) leads to important risk of injuries [1,2]. Indeed, during the whole athletics season, about 61 to 76\% of athletes reported at least one injury [3-5]. In addition, on a total of 14 international athletics championships from 2007 to 2014, a total of 1510 injuries were collected corresponding to an incidence of 100 injuries for 1000 registered athletes [6]. The principal type of injury during international athletics championships was muscle injuries accounting for about $41 \%$ of all injuries [7]. Thigh muscles injuries represented 53\% of muscle injuries and 19\% of all injuries, and hamstring muscles injuries was the first injury diagnosis with $17 \%$ of all injuries [7].

Another important finding was the sex-related difference in injury rates and characteristics between male and female athletes during international athletics championships: male had 25\% higher injury rates than female athletes [6]. The difference was mainly due to the sex-related difference in injury rate of muscle injuries (32 vs. 20 injuries per 1000 athletes for male and female athletes, respectively), muscle injuries representing the most common injury type in both male and female athletes [6]. Moreover, it was the only sex-related different injury type between male and female (with the exception of stress fractures which are less common ( $0.7 \%$ in male and $3.0 \%$ in female athletes)) [6]. More specifically, sex-related difference in injury rate was also mainly due to the sex-related difference in thigh muscles injuries (28 vs. 15 injuries 
per 1000 athletes for male and female athletes, respectively), and especially hamstring muscles injuries (22 vs. 12 injuries per 1000 athletes for male and female athletes, respectively), which were the main injury diagnoses [6].

In this context, a better understanding of sex-related differences in muscle characteristics, especially in thigh muscles characteristics, could help i) to better understand this sex-related difference in the risk of muscle injuries, and ii) to develop injury prevention measures adapted to these specific injury risks. Some parameters of muscle characteristics have been reported to be different between male and female subjects such as muscle body mass, strength, power, or muscle fatigue for instance [8-10]. Although these studies have been performed to describe muscular physiological differences between male and female subjects, these studies could also be of interest to help better explaining sex-related differences in regard to injuries. Therefore, the aim of the present study was to determine, through a systematic review, differences between male and female subjects in the thigh muscles characteristics, separated into architectural (pennation, thickness, and/or fascicle length), mechanical (mass, strength, power, and/or stiffness), neuromuscular (activity) and fatigue aspects, in order to better understand the sex-related differences in the risk of muscle injuries.

\section{Methods}

Search strategy and articles selection

Following the PRISMA guidelines, we conducted a systematic research on MEDLINE via PubMed for all articles analysing comparisons between male and female subjects for the thigh muscles characteristics for all articles published until December 10, 2018. Preliminary searches of articles were conducted from March 2017 to August 2018, and final literature search and articles selection were done the December 10, 2018.

The following key words and equation were used to conduct the searches: («Muscle, Skeletal»[Mesh] AND («Sex Characteristics»[Majr] OR «Sex Factors»[Majr])) AND («muscle architecture» OR «muscle stiffness» OR «Muscle Fibers, Skeletal»[Mesh] OR «Muscle Contraction»[Mesh]) AND «humans»[MeSH Terms] NOT «Aged»[Mesh] Filters: Humans; English; Adult: 19-44 years.

Inclusion criteria were: articles concerning the thigh muscles (i.e. hamstring or quadriceps muscles) in physiological situation, written in English, concerning macroscopic muscle characteristics, such as: architectural, mechanical, neuromuscular and fatigue aspects of the muscle. Exclusion criteria were: articles including subject with histories of muscle pathologies and/or injuries or any pain, and articles about microscopic muscle characteristics such as: myocytes, blood flow, and mitochondrial respiration. After removal of duplicate articles, we selected the articles initially based on the title and abstract, then the full articles were retrieved and reviewed for selection following inclusion and exclusion criteria.

References of selected articles were screened for appropriate articles. Articles selection was independently performed by two authors (PC and PE). Disagreements were resolved by consensus.

Since the present systematic review did not involve human subjects the Ethical Approval or subjects' informed consent was not necessary, but we used data from previous studies, which should have followed the Ethical standard for research on human subjects according to the Declaration of Helsinki. If the latter was not declared in the article, article was not excluded but this was reported in the results. 


\section{Data Extraction}

Data extraction was performed by using a pre-defined data-abstraction sheet, and based on the findings of the preliminary searches. The following data were extracted: purpose of the study, number of subjects per sex, muscle examined, muscle characteristic studied, results and conclusions. The muscle characteristics were separated into architectural (pennation, thickness, and/or fascicle length), mechanical (mass, strength, power, and/or stiffness), and neuromuscular (activity) aspects, in addition the condition of muscle fatigue has also been distinguished.

In order to better highlight the main results of each study, and to highlight the potential differences between male and female subjects if it exists, the results of each parameter were summarised as: "male > female" or "male = female" or "male $<$ female" and the information regarding the significance, and the conclusion regarding the sex-related difference as: "yes" or "moderate" or "no".

\section{Quality Evaluation}

Study quality was independently assessed by one author using the "Checklist for Measuring Quality" [11]. The following cut-off points have been reported to categorise studies by quality with a Methodological Quality Score (MQS): strong (21+), moderate (14-20), fair (7-13) and poor (<7) [11]. This score was not involved as a inclusion/exclusion criteria. 


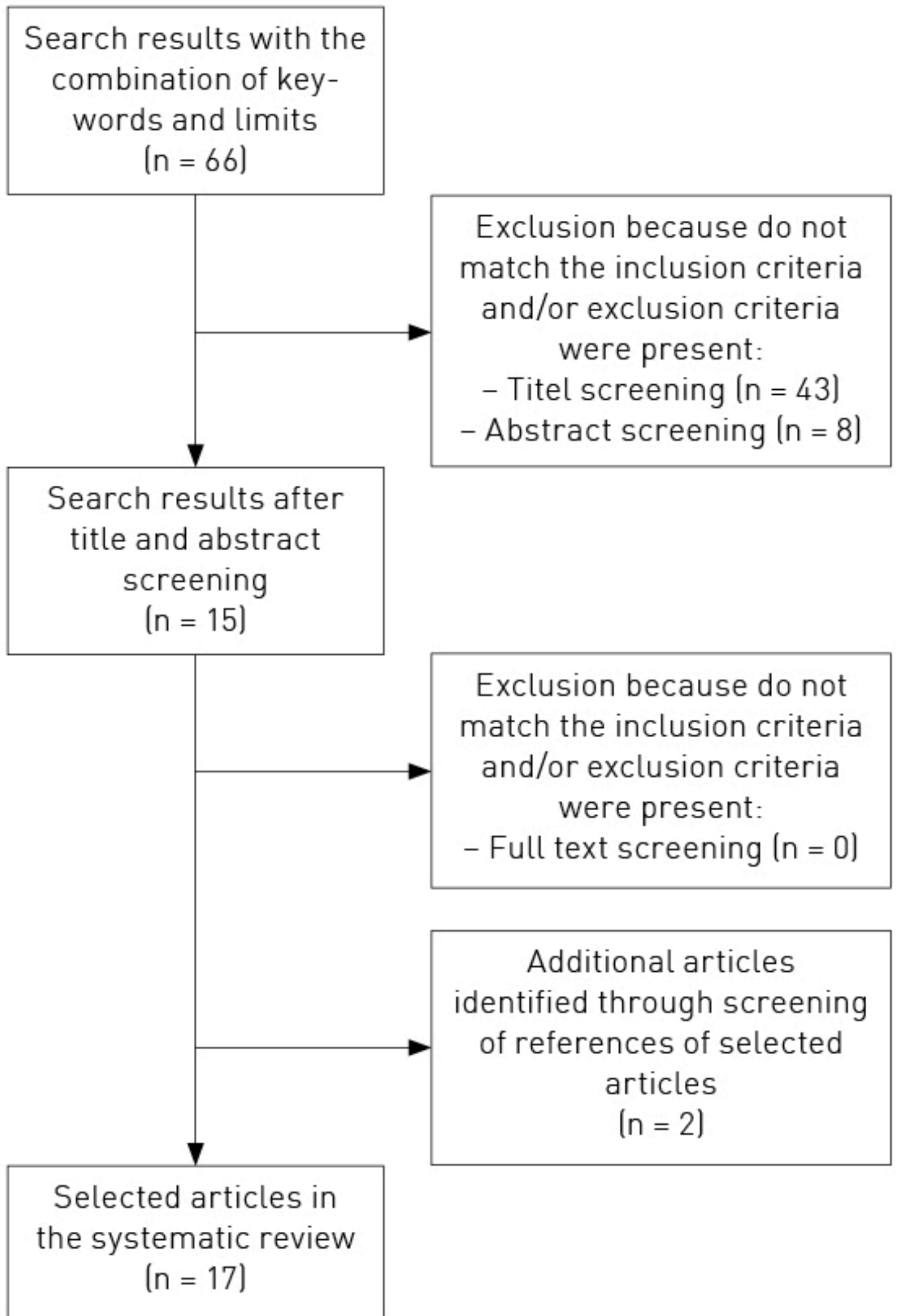

Fig. 1: Flow diagram of the literature screening process and selections of the article. 


\section{Results}

\section{Selected articles}

With the combination of keywords and limits, the literature search revealed 66 articles. 43 articles were excluded after reading their titles and 8 after reading their abstracts because they did not match with the purpose of our present systematic review and/or they did not meet the inclusion criteria. Two articles were find by looking at the references of selected articles. Finally, 17 articles were selected in this review. The flow diagram for the selection of studies is presented in Figure 1.

Articles described different muscle characteristics but they all concerned comparison between sexes on thigh muscles in healthy human subjects. Only one article did not declare that the study followed the Ethical standard for research on human subjects according to the Declaration of Helsinki [12].

\begin{tabular}{|c|c|c|c|c|c|c|c|c|c|}
\hline & $\begin{array}{l}\text { Methodo- } \\
\text { logical } \\
\text { Quality } \\
\text { Score }\end{array}$ & $\begin{array}{l}\text { Number } \\
\text { of males }\end{array}$ & $\begin{array}{l}\text { Number } \\
\text { of } \\
\text { females }\end{array}$ & Muscles & $\begin{array}{l}\text { Aim of the } \\
\text { studies }\end{array}$ & Methods & $\begin{array}{l}\text { Muscle } \\
\text { character- } \\
\text { istics eval- } \\
\text { uated }\end{array}$ & Results & $\begin{array}{l}\text { Conclu- } \\
\text { sions: } \\
\text { Sex-relat- } \\
\text { ed differ- } \\
\text { ences }\end{array}$ \\
\hline $\begin{array}{l}\text { Abe et al. } \\
1998 \text { [13] }\end{array}$ & 17 & 22 & 29 & $\begin{array}{l}\text { Quadri- } \\
\text { ceps } \\
\text { muscle } \\
\text { lvastus } \\
\text { lateralis] }\end{array}$ & $\begin{array}{l}\text { To evaluate } \\
\text { the contribu- } \\
\text { tion of muscle } \\
\text { pennation } \\
\text { angle and } \\
\text { fascicle } \\
\text { length to gen- } \\
\text { der differenc- } \\
\text { es in muscle } \\
\text { size }\end{array}$ & $\begin{array}{l}\text { In vivo mea- } \\
\text { surement of } \\
\text { muscle thick- } \\
\text { ness, penna- } \\
\text { tion angle and } \\
\text { fascicle } \\
\text { length }\end{array}$ & $\begin{array}{l}\text { Muscle } \\
\text { thickness } \\
\text { Pennation } \\
\text { angle } \\
\text { Fascicle } \\
\text { length }\end{array}$ & $\begin{array}{l}\text { Male > } \\
\text { female } \\
\text { Male > } \\
\text { female } \\
\text { Male > } \\
\text { female }\end{array}$ & Yes \\
\hline
\end{tabular}

Table 1: Studies concerning the comparison of thigh muscles architecture between male and female healthy adult subjects.

One article described the muscle architectural characteristics (Table 1) [13], six articles described the muscle mechanical characteristics using the parameters of strength, work, power, torque and/or stiffness measurements

(Table 2) [8-10,14-16], eight articles described neuromuscular characteristics using the parameter of muscle activity (Table 3) [10,17-23], and finally five articles described the sex-related comparisons regarding muscle fatigue (Table 4 )

[8-10,24-26].

\section{Methodological quality}

All thirteen articles were assessed against the Down's and Black checklist and attained a score of either moderate or high quality. Almost all articles reported a high quality score (21+). The main areas of weakness in all articles were their internal validity and power analysis. 


\begin{tabular}{|c|c|c|c|c|c|c|c|c|c|}
\hline & $\begin{array}{l}\text { Methodo- } \\
\text { logical } \\
\text { Quality } \\
\text { Score }\end{array}$ & $\begin{array}{l}\text { Number } \\
\text { of males }\end{array}$ & $\begin{array}{l}\text { Number } \\
\text { of } \\
\text { females }\end{array}$ & Muscles & $\begin{array}{l}\text { Aim of the } \\
\text { studies }\end{array}$ & Methods & $\begin{array}{l}\text { Muscle } \\
\text { character- } \\
\text { istics eval- } \\
\text { uated }\end{array}$ & Results & $\begin{array}{l}\text { Conclu- } \\
\text { sions: } \\
\text { Sex-relat- } \\
\text { ed differ- } \\
\text { ences }\end{array}$ \\
\hline $\begin{array}{l}\text { Pincivero } \\
\text { et al. } 2003 \\
\text { [8] }\end{array}$ & 24 & 19 & 20 & $\begin{array}{l}\text { Quadri- } \\
\text { ceps and } \\
\text { hamstring } \\
\text { muscle }\end{array}$ & $\begin{array}{l}\text { To examine } \\
\text { gender differ- } \\
\text { ences in knee } \\
\text { extensor and } \\
\text { flexor peak } \\
\text { torque, work, } \\
\text { power and } \\
\text { muscle fa- } \\
\text { tigue }\end{array}$ & $\begin{array}{l}30 \text { reciprocal, } \\
\text { concentric } \\
\text { maximal knee } \\
\text { extension and } \\
\text { flexion con- } \\
\text { tractions at a } \\
\text { pre-set angu- } \\
\text { lar }\end{array}$ & $\begin{array}{l}\text { Work, } \\
\text { power and } \\
\text { peak } \\
\text { torque } \\
\end{array}$ & $\begin{array}{l}\text { Male > } \\
\text { female } \\
\text { [signifi- } \\
\text { cant) }\end{array}$ & Yes \\
\hline $\begin{array}{l}\text { Pincivero } \\
\text { et al. } 2003 \\
\text { [14] }\end{array}$ & 24 & 15 & 15 & $\begin{array}{l}\text { Quadri- } \\
\text { ceps } \\
\text { muscle }\end{array}$ & $\begin{array}{l}\text { To compare } \\
\text { perceived } \\
\text { exertion rat- } \\
\text { ings between } \\
\text { males and } \\
\text { females }\end{array}$ & $\begin{array}{l}\text { Evaluation of } \\
\text { the one-repe- } \\
\text { tition maxi- } \\
\text { mum [1-MR] } \\
\text { then two sub- } \\
\text { strate maxi- } \\
\text { mal contrac- } \\
\text { tion in a } \\
\text { random order } \\
\text { at } 20 \% \text { to } 90 \% \\
\text { of their 1-MR }\end{array}$ & Strength & $\begin{array}{l}\text { Male > } \\
\text { female } \\
\text { [signifi- } \\
\text { cant) }\end{array}$ & Yes \\
\hline $\begin{array}{l}\text { Pincivero } \\
\text { et al. } 2004 \\
\text { [15] }\end{array}$ & 24 & 14 & 15 & $\begin{array}{l}\text { Quadri- } \\
\text { ceps } \\
\text { muscle }\end{array}$ & $\begin{array}{l}\text { To examine } \\
\text { gender-spe- } \\
\text { cific knee } \\
\text { extensor } \\
\text { torque output } \\
\text { and quadri- } \\
\text { ceps femoris } \\
\text { muscle re- } \\
\text { cruitment } \\
\text { during MCV }\end{array}$ & $\begin{array}{l}\text { Three iso- } \\
\text { metric con- } \\
\text { traction in a } \\
\text { random order } \\
\text { with the knee } \\
\text { at } 0^{\circ} \text { to } 90^{\circ} \\
\text { levery } 20^{\circ} \text { ] } \\
\text { flexion }\end{array}$ & $\begin{array}{l}\text { Average } \\
\text { torque }\end{array}$ & $\begin{array}{l}\text { Male > } \\
\text { female } \\
\text { [signifi- } \\
\text { cant] }\end{array}$ & Yes \\
\hline $\begin{array}{l}\text { Wüst et } \\
\text { al. } 2008 \\
\text { [9] }\end{array}$ & 23 & 29 & 35 & $\begin{array}{l}\text { Quadri- } \\
\text { ceps } \\
\text { muscle }\end{array}$ & $\begin{array}{l}\text { To examine } \\
\text { the muscle } \\
\text { contractile } \\
\text { properties }\end{array}$ & $\begin{array}{l}\text { MRI [muscle } \\
\text { size), Maximal } \\
\text { voluntary } \\
\text { contractions } \\
\text { (MVC) repeat- } \\
\text { ed two or } \\
\text { three times at } \\
\text { knee joint } \\
\text { angles of } 60 \text {, } \\
70,80 \text { in a } \\
\text { random order }\end{array}$ & $\begin{array}{l}\text { Muscle } \\
\text { size and } \\
\text { strength }\end{array}$ & $\begin{array}{l}\text { Male > } \\
\text { female } \\
\text { [signifi- } \\
\text { cant] }\end{array}$ & Yes \\
\hline $\begin{array}{l}\text { Wüst et } \\
\text { al. } 2008 \\
\text { [9] }\end{array}$ & 23 & 29 & 35 & $\begin{array}{l}\text { Quadri- } \\
\text { ceps } \\
\text { muscle }\end{array}$ & $\begin{array}{l}\text { To examine } \\
\text { the muscle } \\
\text { contractile } \\
\text { properties }\end{array}$ & $\begin{array}{l}\text { MRI (muscle } \\
\text { size), Maximal } \\
\text { voluntary } \\
\text { contractions } \\
\text { (MVC) repeat- } \\
\text { ed two or } \\
\text { three times at } \\
\text { knee joint } \\
\text { angles of } 60 \text {, } \\
70,80 \text { in a } \\
\text { random order }\end{array}$ & Torque & $\begin{array}{l}\text { Female > } \\
\text { Male (not } \\
\text { gignifi- } \\
\text { cant) }\end{array}$ & Moderate \\
\hline
\end{tabular}




\section{Data synthesis}

Concerning muscle architecture, one article reported that isolated muscle thickness and pennation angle of vastus lateralis (quadriceps) were greater in male subjects, and there were no sex-related differences in fascicle length [13].

\begin{tabular}{|c|c|c|c|c|c|c|c|c|c|}
\hline & $\begin{array}{l}\text { Methodo- } \\
\text { logical } \\
\text { Quality } \\
\text { Score }\end{array}$ & $\begin{array}{l}\text { Number } \\
\text { of males }\end{array}$ & $\begin{array}{l}\text { Number } \\
\text { of } \\
\text { females }\end{array}$ & Muscles & $\begin{array}{l}\text { Aim of the } \\
\text { studies }\end{array}$ & Methods & $\begin{array}{l}\text { Muscle } \\
\text { character- } \\
\text { istics eval- } \\
\text { uated }\end{array}$ & Results & $\begin{array}{l}\text { Conclu- } \\
\text { sions: } \\
\text { Sex-relat- } \\
\text { ed differ- } \\
\text { ences }\end{array}$ \\
\hline $\begin{array}{l}\text { De- } \\
\text { schenes } \\
\text { et al. } 2009 \\
\text { [10] }\end{array}$ & 20 & 10 & 10 & $\begin{array}{l}\text { Right } \\
\text { quadri- } \\
\text { ceps } \\
\text { muscle }\end{array}$ & $\begin{array}{l}\text { Different ad- } \\
\text { aptation to } \\
\text { muscle un- } \\
\text { loading by sex } \\
\text { via electro- } \\
\text { myography }\end{array}$ & $\begin{array}{l}\text { Muscle func- } \\
\text { tion was } \\
\text { quantified } \\
\text { during knee } \\
\text { extensions } \\
\text { before and } \\
\text { following } \\
\text { unloading via } \\
\text { electromyo- } \\
\text { graphy during } \\
\text { maximal iso- } \\
\text { metric con- } \\
\text { tractions }\end{array}$ & $\begin{array}{l}\text { Total work, } \\
\text { average } \\
\text { power and } \\
\text { peak } \\
\text { torque }\end{array}$ & $\begin{array}{l}\text { Male> } \\
\text { female } \\
\text { [signifi- } \\
\text { cant] }\end{array}$ & Yes \\
\hline $\begin{array}{l}\text { Wang et } \\
\text { al. } 2015 \\
\text { [16] }\end{array}$ & 21 & 22 & 22 & $\begin{array}{l}\text { Right } \\
\text { quadri- } \\
\text { ceps } \\
\text { muscle }\end{array}$ & $\begin{array}{l}\text { To investigate } \\
\text { knee joints } \\
\text { stiffness be- } \\
\text { tween young } \\
\text { male and } \\
\text { female ath- } \\
\text { letes }\end{array}$ & $\begin{array}{l}\text { Peak torque } \\
\text { of the knee } \\
\text { joint extensor } \\
\text { musculature } \\
\text { was assessed } \\
\text { on an isoki- } \\
\text { netic dyna- } \\
\text { mometer, } \\
\text { muscle stiff- } \\
\text { ness of the } \\
\text { vastus later- } \\
\text { alis muscle } \\
\text { was mea- } \\
\text { sured in both } \\
\text { relaxed and } \\
\text { contracted } \\
\text { conditions }\end{array}$ & $\begin{array}{l}\text { Peak } \\
\text { torque, and } \\
\text { relaxed } \\
\text { and con- } \\
\text { tracted } \\
\text { muscle } \\
\text { stiffness }\end{array}$ & $\begin{array}{l}\text { Male > } \\
\text { female } \\
\text { [signifi- } \\
\text { cant] }\end{array}$ & Yes \\
\hline
\end{tabular}

Concerning the five studies about the muscle mechanical characteristics, all of them reported differences between male and female subjects (Table 2). Two articles $[8,10]$ reported significant higher total work values for male than female subjects (1.76 J.kg-1 for male vs. $1.27 \mathrm{~J} . \mathrm{kg}-1$ for female subjects for knee 
extension and 1.20 J.kg-1 for male and 0.88 J.kg-1 for female subjects $(\mathrm{p}<0.05)$ [8]). These two articles also reported significant higher power values for male than for female subjects ( $5.17 \mathrm{~W}$. kg-1 for male vs. 3.70 W.kg-1 for female subjects for knee extension and 3.52 W.kg-1 for male and 2.59 W.kg-1 for female subjects $(\mathrm{p}<0.05)[8])$. Two articles $[9,14,16]$ reported that male have significantly higher strength values than female subjects. Finally, three of the four studies $[8,10,15,16]$ reported a significant difference between male and female subjects with greater torque values in male subjects. Only one study [9] reported different results with a $4 \%$ higher torque in female subjects compared to male subjects but this did not reach statistical significance $(\mathrm{p}=0.063)$. One article reported higher muscle stiffness values (relaxed and contracted) in male than female subjects [16].

Regarding studies about neuromuscular characteristics (Table 3), results are not consensual. One study [23] did not report any significant difference of total reaction time, pre-motor time and motor time between sexes. Reflex responses [22] may vary between sexes under the "do not innerve" condition. Male subjects demonstrated much more frequent and consistent reflex muscle activation than female subjects. Concerning the effect of unloading, muscle activity seems to be different between sexes after unloading (34\% reduction of the electromyography noted among female and $8 \%$ for male subjects, $p=0.002$ ) [10] but not before. However, one article [21] reported that female subjects had a significant higher level of muscle activity than their male counterparts.

For studies about the muscle fatigue, the four $[8,9,24,26]$ of the five articles reported a difference between male and female subjects (Table 4): male exhibited significant higher fatigue rates than female subjects. 


\begin{tabular}{|c|c|c|c|c|c|c|c|c|c|}
\hline & $\begin{array}{l}\text { Methodo- } \\
\text { logical } \\
\text { Quality } \\
\text { Score }\end{array}$ & $\begin{array}{l}\text { Number } \\
\text { of males }\end{array}$ & $\begin{array}{l}\text { Number } \\
\text { of } \\
\text { females }\end{array}$ & Muscles & $\begin{array}{l}\text { Aim of the } \\
\text { studies }\end{array}$ & Methods & $\begin{array}{l}\text { Muscle } \\
\text { character- } \\
\text { istics eval- } \\
\text { uated }\end{array}$ & Results & $\begin{array}{l}\text { Conclu- } \\
\text { sions: } \\
\text { Sex-relat- } \\
\text { ed differ- } \\
\text { ences }\end{array}$ \\
\hline $\begin{array}{l}\text { Pincivero } \\
\text { et al. } 2000 \\
\text { [17] }\end{array}$ & 24 & 15 & 15 & $\begin{array}{l}\text { quadri- } \\
\text { ceps } \\
\text { muscle }\end{array}$ & $\begin{array}{l}\text { To evaluate } \\
\text { sex and mus- } \\
\text { cle differenc- } \\
\text { es in electro- } \\
\text { myography } \\
\text { amplitude }\end{array}$ & $\begin{array}{l}\text { Electromyo- } \\
\text { graphy acti- } \\
\text { vity during } \\
\text { three maxi- } \\
\text { mal isomet- } \\
\text { ric voluntary } \\
\text { contractions } \\
\text { with the knee } \\
\text { at } 60^{\circ} \text { flexion }\end{array}$ & $\begin{array}{l}\text { Muscle } \\
\text { activity }\end{array}$ & $\begin{array}{l}\text { [signifi- } \\
\text { cant, only } \\
\text { vastus } \\
\text { lateralis } \\
\text { muscle] }\end{array}$ & Moderate \\
\hline $\begin{array}{l}\text { Pincivero } \\
\text { et al. } 2001 \\
\text { [18] }\end{array}$ & 24 & 15 & 15 & $\begin{array}{l}\text { quadri- } \\
\text { ceps } \\
\text { muscle }\end{array}$ & $\begin{array}{l}\text { Electromyo- } \\
\text { graphy dur- } \\
\text { ing isometric } \\
\text { contractions } \\
\text { with the knee } \\
\text { at } 60^{\circ} \text { flexion }\end{array}$ & $\begin{array}{l}\text { Repeated } \\
\text { measure- } \\
\text { ments de- } \\
\text { sign, series } \\
\text { of } 5 \text { s maxi- } \\
\text { mal voluntary } \\
\text { isometric } \\
\text { contractions, } \\
\text { performed at } \\
\text { intensities of } \\
10 \text { to } 90 \% \text { of } \\
\text { MVC }\end{array}$ & $\begin{array}{l}\text { Muscle } \\
\text { activity }\end{array}$ & $\begin{array}{l}\text { Male > } \\
\text { female }\end{array}$ & Moderate \\
\hline $\begin{array}{l}\text { Pincivero } \\
\text { et al. } 2004 \\
\text { [15] }\end{array}$ & 24 & 15 & 15 & $\begin{array}{l}\text { quadri- } \\
\text { ceps } \\
\text { muscle }\end{array}$ & $\begin{array}{l}\text { Electromyo- } \\
\text { graphy dur- } \\
\text { ing isometric } \\
\text { contractions } \\
\text { with the knee } \\
\text { at } 60^{\circ} \text { flexion }\end{array}$ & $\begin{array}{l}\text { Repeated } \\
\text { measure- } \\
\text { ments de- } \\
\text { sign, series } \\
\text { of } 5 \text { s maxi- } \\
\text { mal voluntary } \\
\text { isometric } \\
\text { contractions, } \\
\text { separately } \\
\text { performed in } \\
\text { a random } \\
\text { order }\end{array}$ & $\begin{array}{l}\text { Muscle } \\
\text { activity }\end{array}$ & $\begin{array}{l}\text { Male > } \\
\text { female } \\
\text { (signifi- } \\
\text { cantl) }\end{array}$ & Moderate \\
\hline $\begin{array}{l}\text { Cramer et } \\
\text { al. } 2002 \\
\text { [20] }\end{array}$ & 23 & 11 & 12 & $\begin{array}{l}\text { quadri- } \\
\text { ceps } \\
\text { muscle }\end{array}$ & $\begin{array}{l}\text { To explore } \\
\text { the sex, mus- } \\
\text { cle and ve- } \\
\text { locity re- } \\
\text { sponses }\end{array}$ & $\begin{array}{l}\text { Concentric, } \\
\text { isokinetic } \\
\text { peal torque } \\
\text { for extension } \\
\text { of the domi- } \\
\text { nant leg was } \\
\text { measured at } \\
\text { randomly } \\
\text { order velo- } \\
\text { cities of } 60 \text { to } \\
300^{\circ} \text { levery } \\
60 \text { ] }\end{array}$ & $\begin{array}{l}\text { Electro- } \\
\text { myography } \\
\text { amplitudes }\end{array}$ & $\begin{array}{l}\text { sex- and } \\
\text { muscle- } \\
\text { specific } \\
\text { lonly } 2 \\
\text { muscle in } \\
\text { female] }\end{array}$ & Moderate \\
\hline $\begin{array}{l}\text { DeMont et } \\
\text { al. } 2004 \\
\text { [21] }\end{array}$ & 19 & 17 & 17 & $\begin{array}{l}\text { hamstring } \\
\text { muscle }\end{array}$ & $\begin{array}{l}\text { To determine } \\
\text { if the level of } \\
\text { preactivation } \\
\text { of hamstring } \\
\text { muscles dur- } \\
\text { ing dynamic } \\
\text { activity is } \\
\text { affected by } \\
\text { sex }\end{array}$ & $\begin{array}{l}\text { Maximum } \\
\text { voluntary } \\
\text { contraction } \\
\text { of the quadri- } \\
\text { ceps and } \\
\text { hamstrings } \\
\text { muscles dur- } \\
\text { ing downhill } \\
\text { walking and } \\
\text { running }\end{array}$ & $\begin{array}{l}\text { Muscle } \\
\text { activity }\end{array}$ & $\begin{array}{l}\text { Female > } \\
\text { male [sig- } \\
\text { nificant] }\end{array}$ & Yes \\
\hline
\end{tabular}




\begin{tabular}{|c|c|c|c|c|c|c|c|c|c|}
\hline & $\begin{array}{l}\text { Methodo- } \\
\text { logical } \\
\text { Quality } \\
\text { Score }\end{array}$ & $\begin{array}{l}\text { Number } \\
\text { of males }\end{array}$ & $\begin{array}{l}\text { Number } \\
\text { of } \\
\text { females }\end{array}$ & Muscles & $\begin{array}{l}\text { Aim of the } \\
\text { studies }\end{array}$ & Methods & $\begin{array}{l}\text { Muscle } \\
\text { character- } \\
\text { istics eval- } \\
\text { uated }\end{array}$ & Results & $\begin{array}{l}\text { Conclu- } \\
\text { sions: } \\
\text { Sex-relat- } \\
\text { ed differ- } \\
\text { ences }\end{array}$ \\
\hline $\begin{array}{l}\text { Deschenes } \\
\text { et al. } 2009 \\
\text { [10] }\end{array}$ & 20 & 10 & 10 & $\begin{array}{l}\text { right knee } \\
\text { muscle }\end{array}$ & $\begin{array}{l}\text { To explore } \\
\text { whether male } \\
\text { and female } \\
\text { experienced } \\
\text { different ad- } \\
\text { aptation to } \\
\text { muscle } \\
\text { unloading }\end{array}$ & $\begin{array}{l}\text { Muscle func- } \\
\text { tion was } \\
\text { quantified } \\
\text { during knee } \\
\text { extensions } \\
\text { before and } \\
\text { following } \\
\text { unloading via } \\
\text { electromyo- } \\
\text { graphy dur- } \\
\text { ing maximal } \\
\text { isometric } \\
\text { contractions }\end{array}$ & $\begin{array}{l}\text { Muscle } \\
\text { activity }\end{array}$ & $\begin{array}{l}\text { Female > } \\
\text { male (sig- } \\
\text { nificant]. } \\
\text { Female > } \\
\text { male in } \\
\text { decline of } \\
\text { EMG (sig- } \\
\text { nificant] }\end{array}$ & Moderate \\
\hline $\begin{array}{l}\text { Cammara- } \\
\text { ta et al. } \\
2010 \text { [22] }\end{array}$ & 22 & 12 & 12 & $\begin{array}{l}\text { quadri- } \\
\text { ceps and } \\
\text { hamstring } \\
\text { muscle }\end{array}$ & $\begin{array}{l}\text { To explore } \\
\text { the effect on } \\
\text { volitional and } \\
\text { reflex neuro- } \\
\text { muscular } \\
\text { responses to } \\
\text { a rapid val- } \\
\text { gus pertur- } \\
\text { bation }\end{array}$ & $\begin{array}{l}\text { multiple } 7^{\circ} \\
\text { ramp-and- } \\
\text { hold valgus } \\
\text { perturba- } \\
\text { tions, in } \\
\text { "innerve } \\
\text { condition" } \\
\text { (resistance) } \\
\text { and "do not } \\
\text { innerve"- }\end{array}$ & $\begin{array}{l}\text { Reflex } \\
\text { responses }\end{array}$ & $\begin{array}{l}\text { under "do } \\
\text { not in- } \\
\text { nerve": } \\
\text { Male> } \\
\text { female } \\
\text { (signifi- } \\
\text { cant), } \\
\text { under } \\
\text { "innerve": } \\
\text { no differ- } \\
\text { ence }\end{array}$ & Moderate \\
\hline $\begin{array}{l}\text { Ayala et al. } \\
2004 \text { [23] }\end{array}$ & 24 & 24 & 24 & $\begin{array}{l}\text { hamstring } \\
\text { muscle }\end{array}$ & $\begin{array}{l}\text { To examine } \\
\text { sex-related } \\
\text { differences in } \\
\text { the ham- } \\
\text { string reac- } \\
\text { tion times } \\
\text { profile }\end{array}$ & $\begin{array}{l}\text { six maximal } \\
\text { voluntary } \\
\text { eccentric } \\
\text { knee flexion } \\
\text { muscle ac- } \\
\text { tions were } \\
\text { performed } \\
\text { with } 30 \text { sec } \\
\text { rest between } \\
\text { each con- } \\
\text { tractions }\end{array}$ & $\begin{array}{l}\text { Total } \\
\text { Reaction, } \\
\text { Pre-Motor } \\
\text { \& Motor } \\
\text { Time }\end{array}$ & $\begin{array}{l}\text { Female > } \\
\text { Male (no } \\
\text { signifi- } \\
\text { cant) }\end{array}$ & No \\
\hline
\end{tabular}

\section{Discussion}

The main findings of the present study were that studies reported higher values of muscle mechanical characteristics for male than female subjects, higher muscle fatigue for male than female subjects, while 
neuromuscular characteristics remains unclear. The vast majority of the studies included focused on the quadriceps muscles, consequently very few is known regarding the hamstring muscles which represented the first injury diagnosis in international athletics championships [7]. 


\begin{tabular}{|c|c|c|c|c|c|c|c|c|c|}
\hline & $\begin{array}{l}\text { Methodo- } \\
\text { logical } \\
\text { Quality } \\
\text { Score }\end{array}$ & $\begin{array}{l}\text { Number } \\
\text { of males }\end{array}$ & $\begin{array}{l}\text { Number } \\
\text { of } \\
\text { females }\end{array}$ & Muscles & $\begin{array}{l}\text { Aim of the } \\
\text { studies }\end{array}$ & Methods & $\begin{array}{l}\text { Muscle } \\
\text { character- } \\
\text { istics eval- } \\
\text { uated }\end{array}$ & Results & $\begin{array}{l}\text { Conclu- } \\
\text { sions: } \\
\text { Sex-relat- } \\
\text { ed differ- } \\
\text { ences }\end{array}$ \\
\hline $\begin{array}{l}\text { Pincivero } \\
\text { et al. } 2003 \\
\text { [8] }\end{array}$ & 24 & 19 & 20 & $\begin{array}{l}\text { quadri- } \\
\text { ceps and } \\
\text { hamstring } \\
\text { muscle }\end{array}$ & $\begin{array}{l}\text { to examine } \\
\text { sex differenc- } \\
\text { es in muscle } \\
\text { fatigue }\end{array}$ & $\begin{array}{l}30 \text { reciprocal } \\
\text { MCV, Muscle } \\
\text { fatigue rate } \\
\text { calculated as } \\
\text { the decline in } \\
\text { each isokinet- } \\
\text { ic variable by } \\
\text { the linear } \\
\text { slope from } \\
\text { the single } \\
\text { highest repe- } \\
\text { tition value } \\
\text { through the } \\
\text { 30th }\end{array}$ & $\begin{array}{l}\text { muscle } \\
\text { fatigue }\end{array}$ & $\begin{array}{l}\text { Male > } \\
\text { female } \\
\text { [gignifi- } \\
\text { cant] }\end{array}$ & Yes \\
\hline $\begin{array}{l}\text { Wüst et } \\
\text { al. [9] }\end{array}$ & 23 & 29 & 35 & $\begin{array}{l}\text { quadri- } \\
\text { ceps } \\
\text { muscle }\end{array}$ & $\begin{array}{l}\text { To determine } \\
\text { whether there } \\
\text { are differenc- } \\
\text { es in suscep- } \\
\text { tibility to pe- } \\
\text { ripheral } \\
\text { fatigue in } \\
\text { male and } \\
\text { female }\end{array}$ & $\begin{array}{l}\text { MVC repeated } \\
\text { two or three } \\
\text { times at knee } \\
\text { joint angles, } \\
\text { Five minutes } \\
\text { after, resis- } \\
\text { tance to fa- } \\
\text { tigue deter- } \\
\text { mined by a } \\
\text { series of } \\
\text { electrically } \\
\text { evoked iso- } \\
\text { metric con- } \\
\text { tractions of } \\
60,70,80 \text { in a } \\
\text { random order }\end{array}$ & \begin{tabular}{|l|} 
muscle \\
fatigue
\end{tabular} & $\begin{array}{l}\text { Male > } \\
\text { female } \\
\text { [signifi- } \\
\text { cant] }\end{array}$ & Yes \\
\hline $\begin{array}{l}\text { Ansdell et } \\
\text { al. } 2017 \\
{[24]}\end{array}$ & 24 & 10 & 8 & $\begin{array}{l}\text { quadri- } \\
\text { ceps } \\
\text { muscle }\end{array}$ & $\begin{array}{l}\text { To determine } \\
\text { the sex differ- } \\
\text { ence in per- } \\
\text { formance } \\
\text { fatigability for } \\
\text { intermittent, } \\
\text { isometric } \\
\text { contractions } \\
\text { of the } \\
\text { knee-exten- } \\
\text { sor muscles }\end{array}$ & $\begin{array}{l}30 \% \text { of MVC } \\
\text { for } 30 \text { min, } \\
\text { and in a sepa- } \\
\text { rate session } \\
\text { at } 50 \% \text { MVC } \\
\text { until } \\
\text { task-failure, } \\
\text { with mea- } \\
\text { surements of } \\
\text { MVC every } 60 \\
\text { sec and EMG } \\
\text { recording } \\
\text { during all } \\
\text { contractions }\end{array}$ & $\begin{array}{l}\text { muscle } \\
\text { fatigue }\end{array}$ & $\begin{array}{l}\text { Male > } \\
\text { female } \\
\text { [signifi- } \\
\text { cant] }\end{array}$ & Yes \\
\hline $\begin{array}{l}\text { Boccia et } \\
\text { al. } 2018 \\
\text { [25] }\end{array}$ & 24 & 10 & 8 & $\begin{array}{l}\text { quadri- } \\
\text { ceps } \\
\text { muscle }\end{array}$ & $\begin{array}{l}\text { To investigate } \\
\text { sex differenc- } \\
\text { es in central } \\
\text { and peripher- } \\
\text { al fatigue and } \\
\text { in rate of } \\
\text { force devel- } \\
\text { opment in the } \\
\text { knee exten- } \\
\text { sors after a } \\
\text { half-mara- } \\
\text { thon run }\end{array}$ & $\begin{array}{l}\text { Knee exten- } \\
\text { sors forces } \\
\text { were obtained } \\
\text { under volun- } \\
\text { tary and elec- } \\
\text { trically } \\
\text { evoked iso- } \\
\text { metric con- } \\
\text { tractions. }\end{array}$ & $\begin{array}{l}\text { central and } \\
\text { peripheral } \\
\text { muscle } \\
\text { fatigue }\end{array}$ & $\begin{array}{l}\text { Male }= \\
\text { female }\end{array}$ & No \\
\hline
\end{tabular}




\begin{tabular}{|c|c|c|c|c|c|c|c|c|c|}
\hline & $\begin{array}{l}\text { Methodo- } \\
\text { logical } \\
\text { Quality } \\
\text { Score }\end{array}$ & $\begin{array}{l}\text { Number } \\
\text { of males }\end{array}$ & $\begin{array}{l}\text { Number } \\
\text { of } \\
\text { females }\end{array}$ & Muscles & $\begin{array}{l}\text { Aim of the } \\
\text { studies }\end{array}$ & Methods & $\begin{array}{l}\text { Muscle } \\
\text { character- } \\
\text { istics eval- } \\
\text { uated }\end{array}$ & Results & $\begin{array}{l}\text { Conclu- } \\
\text { sions: } \\
\text { Sex-relat- } \\
\text { ed differ- } \\
\text { ences }\end{array}$ \\
\hline $\begin{array}{l}\text { O'Leary et } \\
\text { al. } 2018 \\
{[26]}\end{array}$ & 24 & 23 & 19 & $\begin{array}{l}\text { quadri- } \\
\text { ceps } \\
\text { muscle }\end{array}$ & $\begin{array}{l}\text { To investigate } \\
\text { the sex differ- } \\
\text { ence in neu- } \\
\text { romuscular } \\
\text { fatigue of } \\
\text { British Army } \\
\text { recruites }\end{array}$ & $\begin{array}{l}\text { Isometric } \\
\text { maximal vol- } \\
\text { untary con- } \\
\text { traction } \\
\text { (MVC) force of } \\
\text { the knee ex- } \\
\text { tensors were } \\
\text { examined } \\
\text { pre- and } \\
\text { post-loaded } \\
\text { march to } \\
\text { examine neu- } \\
\text { romuscular } \\
\text { fatigue }\end{array}$ & $\begin{array}{l}\text { muscle } \\
\text { fatigue }\end{array}$ & $\begin{array}{l}\text { Male > } \\
\text { female } \\
\text { [signifi- } \\
\text { cant] }\end{array}$ & Yes \\
\hline
\end{tabular}

\section{Sex-related differences in thigh muscles architectural aspects}

Only one study reported results concerning sex-related comparison on muscle thickness, pennation angle and fascicle length, which was published 20 years ago. The results reported that isolated muscle thickness and pennation angle of quadriceps were greater in male than female subjects, and there were no sexrelated differences in fascicle length. Given the fact that this is the only one study and the interest of such parameters in injury occurrence and thus prevention [27], it is of interest to analyse such parameters in future studies, in order to better understand the sex-related difference in thigh (and especially hamstring) injury risk [6].

Sex-related differences in thigh muscles mechanical aspects

These results suggested that thigh muscles, and especially quadriceps muscles, differed between male and female subjects regarding work, power and strength. Despite the few number of studies concerning muscle size and muscle mass, all studies included converged on the same direction with higher strength, work or power rates in male than in female subjects; results are consensual and consistent [8-10,14,15]. Male subjects presented a higher ability to generate force independent of their body mass [14]. Male subjects possessed an inherent ability to generate higher levels of torque than female subjects [8]. Gender-specific patterns in muscle activation and muscle fiber morphology have been reported as viable explanations for these strength differences [14]. Neural factors might explain these differences too. Sex-related disparities observed in the capacity of the nervous system to activate contracting muscle may play a role in sexrelated differences concerning strength, especially after unloading. Female subjects suffered a greater degree of neuromuscular disturbance than men [10]. Regarding injury risk, does this capability to develop 
higher strength can make male athletes at higher risk of muscle injuries than female subjects, because they can outmatch the muscle capability. In addition, sex-related differences seem to exist regarding stiffness [16]. Can this higher stiffness in male than female subjects explain higher sprint performance but higher thigh (and especially hamstring) injuries in males athletes? Future studies should analyse this parameters in comparison to injury occurrence in order to improve understanding on injury risk and help for injury prevention.

\section{Sex-related differences in muscle activity}

The results are different according to the studies. It seems that there is no difference in the intensity or ability to activate the quadriceps muscles [22]. Female may require a greater amount of time to reach a specified percentage of maximal force [23]. Even if non-significant, female subjects reported consistently longer hamstring total reaction time, pre-motor time and motor time and differences in neuromechanical function of the hamstrings [23]. Moreover, female subjects reflex responses are reported to be less frequent and consistent than male's ones [22]. Sex-related differences in neuromuscular control and neurophysiological mechanisms of the lower extremity have been reported to contribute to increase injury risk [22]. However, given the inconsistency of results and the lack of studies analysing the aspects in comparison to injury occurrence, no conclusion could be done, which would have been of interest in an injury prevention perspective.

\section{Sex-related differences in muscle fatigability}

Female subjects appeared to experience muscle fatigue at a slower rate [8]. Intrinsic sex-related differences in skeletal muscle properties, such as contractile speed and rate of energy utilisation, probably play a key role in these sex-related differences in fatigue [9]. Female subjects have slower contractile speed than male subjects [9]. Moreover, the difference in fatiguability may be a consequence of higher energy utilization in male subjects, which is the reason why they may fatigue more rapidly. It must reside in the rate at which energy is used by the muscle fibers rather than the oxydative recovery rates [9]. Male subjects exhibit a higher susceptibility to muscle fatigue and the higher rate of muscle injuries can be related to this susceptibility. This aspect could play a role in the higher thigh muscles injury risk of male aspects, since muscle injuries have been reported to be increased in fatigue condition [28,29]. Female athletes could be better protected against injuries because their muscle kept higher percentage of maximal strength longer than male athletes. This hypothesis should of course be confirmed throught experimental study.

\section{Limitations}

Some limitations have to be discussed. First, only one data base was screened (MEDLINE via PubMed), the present systematic review can thus not be considered as exhaustive. However, this is the database that brings together the most articles on the physiological and medical fields. Second, the quality of the present review depends on the quality of selected studies. Third, despite of the models quite similar between studies, muscle characteristics studied and methods for analyses were different between the selected studies, that makes the comparison difficult. Only some characteristics of the muscle have been analysed representing mechanical and neuromuscular aspects of the muscle. Fourth, other studies reported results regarding sex-related muscle characteristics but involving other muscles than thigh muscles. Finally, many 
muscle characteristics have been presented in these articles, however it could be not exhaustive enough to describe all the all muscle characteristics (potential limitation due to keywords...). Moreover, the analysis is not focused enough on main aspects to settle on precise conclusions. Therefore, further more detailed researches are required.

\section{Perspectives}

Muscle injury has been reported to be the only injury type concerned by sex-differences during international athletics championships [6]. The different studies included in our systematic review reported sex-related differences in the muscle characteristics of thigh muscles: higher levels of power, work, strenght, stiffness and fatibability in male than female subjects. These sex-related differences in muscle characteristics could be involved in the hypothesis to explain the higher muscle injury rate reported in male than in female athletes, but none of these articles can strongly explain the sex-related injury difference. Throughought this systematic review, we cannot conclude on the reason of the higher muscle injury rate in male athletes. However, it gives opportunities for further studies.

\section{Conclusions}

The main findings of the present study were that muscle architecture and mechanical characteristics were higher for male than female subjects, muscle fatigue higher for male than female subjects, while neuromuscular characteristics remains unclear. Although several macroscopic muscle characteristics have been reported to be different between male and female healthy adult subjects, it is difficult to conclude on its exact relationship with the higher muscle injury rates reported in male athletes during international athletics championships.

\section{Conflict of interest}

The authors declare no conflicts of interest.

\section{Corresponding author}

Pascal Edouard, MD PhD, Department of Clinical and

Exercise Physiology, Sports medicine unity, IRMIS, Campus Santé Innovations, University Hospital of Saint-Etienne, 42055 Saint-Etienne cedex 2, France. Tel.: +33 674574 691; fax: +33 477127229

E-mail address:

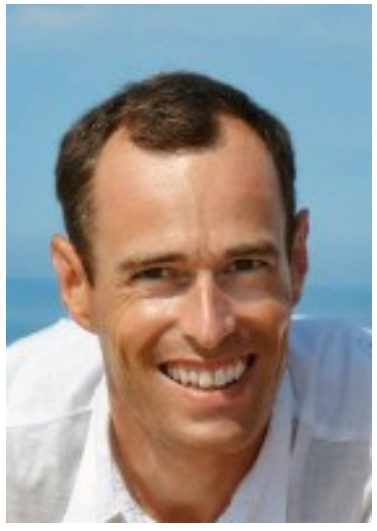

Pascal.Edouard42@gmail.com 


\section{Referenzen}

1. Edouard P, Morel N, Serra J-M, et al. [Prévention des lésions de l'appareil locomoteur liées à la pratique de l'athlétisme sur piste. Revue des données épidémiologiques]. Sci Sports 2011;26:307-15. doi: 10.1016/j.scispo.2011.04.003.

2. Edouard P, Alonso JM, Jacobsson J, et al. Injury Prevention in Athletics: The Race Has Started and We Are on Track! New Stud Athl 2015;30:69-78.

3. D’Souza D. Track and field athletics injuries - a one-year survey. Br J Sports Med 1994;28:197-202.

4. Bennell KL, Crossley K. Musculoskeletal injuries in track and field: Incidence, distribution and risk factors. Aust J Sci Med Sport 1996;28:69-75.

5. Jacobsson J, Timpka T, Kowalski J, et al. Injury patterns in Swedish elite athletics: annual incidence, injury types and risk factors. Br J Sports Med 2013;47:941-52. doi:10.1136/bjsports-2013-092676.

6. Edouard P, Feddermann-Demont N, Alonso JM, et al. Sex differences in injury during top-level international athletics championships: surveillance data from 14 championships between 2007 and 2014. Br J Sport Med 2015;49:472-7. doi:10.1136/bjsports-2014-094316.

7. Edouard P, Branco P, Alonso J-M. Muscle injury is the principal injury type and hamstring muscle injury is the first injury diagnosis during top-level international athletics championships between 2007 and 2015. Br J Sports Med 2016;50:619-30. doi:10.1136/bjsports-2015- 095559.

8. Pincivero DM, Gandaio CMB, Ito Y. Gender-specific knee extensor torque, flexor torque, and muscle fatigue responses during maximal effort contractions. Eur J Appl Physiol 2003;89:134-41. doi:10.1007/s00421-002-0739-5.

9. Wüst RCI, Morse CI, de Haan A, et al. Sex differences in contractile properties and fatigue resistance of human skeletal muscle. Exp Physiol 2008;93:843-50. doi:10.1113/expphysiol.2007.041764.

10. Deschenes MR, McCoy RW, Holdren AN, et al. Gender influences neuromuscular adaptations to muscle unloading. Eur J Appl Physiol 2009;105:889-97. doi:10.1007/s00421-008-0974-5.

11. Downs SH, Black N. The feasibility of creating a checklist for the assessment of the methodological quality both of randomised and non-randomised studies of health care interventions. J Epidemiol Community Heal 1998;52:377-84.

12. DeMont RG, Lephart SM. Effect of sex on preactivation of the gastrocnemius and hamstring muscles. Br J Sports Med 2004;38:120-4. doi:10.1136/bjsm.2003.000195.

13. Abe T, Brechue WF, Fujita S, et al. Gender differences in FFM accumulation and architectural characteristics of muscle. Med Sci Sports Exerc 1998;30:1066-70.

14. Pincivero DM, Coelho AJ, Campy RM. Perceived exertion and maximal quadriceps femoris muscle strength during dynamic knee extension exercise in young adult males and females. Eur J Appl Physiol 2003;89:150-6. doi:10.1007/s00421-002-0768-0.

15. Pincivero DM, Salfetnikov Y, Campy RM, et al. Angle- and gender- specific quadriceps femoris muscle recruitment and knee extensor torque. J Biomech 2004;37:1689-97. doi:10.1016/j.jbiomech.2004. 02.005 .

16. Wang D, De Vito G, Ditroilo M, et al. A comparison of muscle stiffness and musculoarticular stiffness of the knee joint in young athletic males and females. J Electromyogr Kinesiol 2015;25:495-500. doi: 10.1016/j.jelekin.2015.03.003.

17. Pincivero DM, Green RC, Mark JD, et al. Gender and muscle differences in EMG amplitude and median frequency, and variability during maximal voluntary contractions of the quadriceps femoris. J Electromyogr Kinesiol Off J Int Soc Electrophysiol Kinesiol 2000;10:189-96.

18. Pincivero DM, Campy RM, Salfetnikov Y, et al. Influence of contraction intensity, muscle, and gender on median frequency of the quadriceps femoris. J Appl Physiol (Bethesda, Md 1985) 2001;90:804-10.

19. Pincivero DM, Coelho AJ, Campy RM, et al. The effects of voluntary contraction effort on quadriceps femoris electromyogram median frequency in humans: a muscle and sex comparison. Eur J Appl Physiol 2002;87:448-55. doi:10.1007/s00421-002-0658-5. 
20. Cramer JT, Housh TJ, Weir JP, et al. Power output, mechanomyographic, and electromyographic responses to maximal, concentric, isokinetic muscle actions in men and women. J Strength Cond Res 2002; 16:399-408.

21. DeMont RG, Lephart SM. Effect of sex on preactivation of the gastrocnemius and hamstring muscles. Br J Sports Med 2004;38:120-4.

22. Cammarata ML, Dhaher YY. Evidence of gender-specific motor templates to resist valgus loading at the knee. Muscle Nerve 2010;41:614-23. doi:10.1002/mus.21509.

23. Ayala F, De Ste Croix M, Sainz de Baranda P, et al. Inter-session reliability and sex-related differences in hamstrings total reaction time, pre-motor time and motor time during eccentric isokinetic contractions in recreational athlete. J Electromyogr Kinesiol Off J Int Soc Electrophysiol Kinesiol 2014;24:200-6. doi:10.1016/j.jelekin.2013. 12.006.

24. Ansdell P, Thomas K, Howatson G, et al. Contraction intensity and sex differences in knee-extensor fatigability. J Electromyogr Kinesiol 2017;37:68-74. doi:10.1016/j.jelekin.2017.09.003.

25. Boccia G, Dardanello D, Tarperi C, et al. Women show similar central and peripheral fatigue to men after half-marathon*. Eur J Sport Sci 2018;18:695-704. doi:10.1080/17461391.2018.1442500

26. O'Leary TJ, Saunders SC, McGuire SJ, et al. Sex differences in neuromuscular fatigability in response to load carriage in the field in British Army recruits. J Sci Med Sport 2018;21:591-5. doi:10.1016/j.jsams.2017.10.018.

27. Timmins RG, Bourne MN, Shield AJ, et al. Short biceps femoris fascicles and eccentric knee flexor weakness increase the risk of hamstring injury in elite football (soccer): a prospective cohort study. Br J Sports Med 2016;50:1524-35. doi:10.1136/bjsports-2015-095362.

28. Woods C, Hawkins RD, Maltby S, et al. The Football Association Medical Research Programme: an audit of injuries in professional football-analysis of hamstring injuries. Br J Sport Med 2004;38:36-41. doi:10.1136/bjsm.2002.002352.

29. Brooks JHM, Fuller CW, Kemp SPT, et al. Incidence, Risk, and Prevention of Hamstring Muscle Injuries in Professional Rugby Union. Am J Sports Med 2006;34:1297-306. doi:10.1177/0363546505286022. 